\title{
PSICOPATOLOGIAS NA INFÂNCIA E NA ADOLESCÊNCIA
}

\section{PSYCHOPATHOLOGIES IN CHILDHOOD AND ADOLESCENCE}

\author{
Camilla Casotti Poisk ${ }^{*}$, Elisa Ávila Casotti Poisk ${ }^{1}$, Julia Fernanda Santos Miotto \\ Vagner Fagnani Linartevichi ${ }^{1}$ \\ ${ }^{1}$ Curso de Psicologia do Centro Universitário Fundação Assis Gurgacz - FAG
}

Autor correspondente: ccpoisk@gmail.com , https://orcid.org/0000-0002-2624-7744

\begin{abstract}
RESUMO
O presente artigo se constitui em uma revisão bibliográfica sobre transtornos mentais em crianças e adolescentes e seus respectivos tratamentos farmacológicos, abrangendo três psicopatologias da infância e da adolescência: o Transtorno de Déficit de Atenção/Hiperatividade, Transtornos de Ansiedade e Transtornos Disruptivos, do Controle de Impulsos e da Conduta. O Transtorno de Déficit de Atenção/Hiperatividade, é um transtorno do neurodesenvolvimento, caracterizado por três subtipos, considerando a ocorrência nos últimos seis meses, e o tratamento farmacológico abarca, principalmente, o uso de metilfenidato. Os Transtornos de Ansiedade englobam onze transtornos mentais, nos quais o medo e a ansiedade demarcam como características fundamentais, sendo sua farmacoterapia de classe dos ISRS, antidepressivos tricíclicos e benzodiazepínicos. Somando com os transtornos citados, os Transtornos Disruptivos, do Controle de Impulsos e da Conduta, conceituam-se categoricamente por extrema dificuldade em lidar e ter controle das emoções e comportamentos, causando, imprescindivelmente, intenso sofrimento ao sujeito e prejuízos sociais, como a perda de liberdade e penalizações de violação de normas e leis, sendo que a indicação terapêutica é composta pelo uso de ácido valpóico, lítio, risperidona ou carbamazepina.
\end{abstract}

Palavras-chave: Psicopatologia, Transtornos Mentais, Diagnóstico.

\begin{abstract}
This article is a literature review on mental disorders in children and adolescents and their respective pharmacological treatments, covering three psychopathologies of childhood and adolescence: Attention Deficit / Hyperactivity Disorder, Anxiety Disorders and Disruptive Control Disorders. Impulses and Conduct. Attention Deficit / Hyperactivity Disorder is a neurodevelopmental disorder, characterized by three subtypes, considering its occurrence in the last six months, and the pharmacological treatment includes mainly the use of methylphenidate. Anxiety Disorders include eleven mental disorders, in which fear and anxiety demarcate as fundamental characteristics, being their SSRI-class pharmacotherapy, tricyclic antidepressants and benzodiazepines. In addition to the aforementioned disorders, Disruptive, Impulse Control and Conduct Disorders are categorically conceptualized as extremely difficult to deal with and have control of emotions and behaviors, causing, essentially, intense suffering to the subject and social damage, such as loss. freedom and penalties for violation of rules and laws, and the therapeutic indication is the use of valpoic acid, lithium, risperidone or carbamazepine.
\end{abstract}

Keywords: Psychopathology, Mental Disorders, Diagnosis. 


\section{INTRODUÇÃO}

O presente artigo busca descrever a partir de considerações sobre 0 desenvolvimento e o ciclo vital, aspectos e critérios de psicopatologias, sendo definidos em relação a normas, valores culturais, sociais e familiares, considerando transtorno mental como utilidade clínica que objetiva aos clínicos determinar um prognóstico, planos de tratamento e possíveis resultados, norteados conforme gravidade dos sintomas, sua importância e sofrimento que causa no indivíduo (APA, 2014).

Os transtornos mentais que se configuram, dentre os mais prevalentes na infância e na adolescência, concordantemente com as diretrizes do Manual Estatístico de Transtornos Mentais - DSM-5, e CID 10, retratados nesse artigo, são o Transtorno de Déficit de Atenção, Transtornos de Ansiedade e os Transtornos Disruptivos, do Controle de Impulsos e da Conduta, cuja clínica possui suas particularidades por se tratar de acompanhar as primeiras fases do desenvolvimento humano, e que serão responsáveis pela precisão diagnóstica, sua precocidade em avaliação, conhecimento e compreensão de sintomas e a evolução dos mesmos, considerando que esses fatores são fundamentais para eficácia do tratamento (TISSER, 2018).

Salienta-se, então, que o conceito de normalidade e patologia, traz grandes controvérsias em profissionais da área da saúde mental, e o limite entre o normal e o patológico é impreciso, diante a subjetividade do indivíduo, uma vez que dois corpos podem ter a mesma influência e reagirem distintamente. Logo, segundo Caudilhem (2010), é para além do corpo que se deve olhar para se julgar o normal e o patológico para este mesmo corpo, tornando-se necessário um olhar além de partes do corpo, ou sua totalidade, pois existe um mal-estar, que abrange um além do limite do corpo, que se encontra na sociedade.

\section{METODOLOGIA}

Este artigo foi elaborado em formato de pesquisa bibliográfica, sendo esta fundamentada em obras de diversos autores contemporâneos, publicadas em livros, artigos científicos e revistas, disponíveis eletronicamente (Scielo) e em bibliotecas.

\section{TRANSTORNO DE DÉFICIT DE ATENÇÃO/HIPERATIVIDADE}

O Transtorno de Déficit de Atenção/Hiperatividade, popularmente conhecido como TDAH, é definido, de acordo com o DSM-5 (APA, 2014), pela perseverante presença de desatenção e/ou hiperatividade que destoa do esperado para a fase do desenvolvimento em que a pessoa se encontra, acarretando significativo prejuízo funcional. Ele tem início na infância e seus sintomas devem se fazer presentes em mais de um ambiente.

Para o diagnóstico, segundo Whitbourne e Halgin (2015), é de suma relevância distinguir os três subtipos desse transtorno. Declara-se que o TDAH é do "tipo combinado" quando o critério de desatenção é preenchido juntamente com o critério de hiperatividade. Já o TDAH do "tipo predominantemente desatento" é diagnosticado quando apenas o critério para desatenção é satisfeito. Enquanto o terceiro subtipo, "tipo predominantemente hiperativo/impulsivo", se refere ao preenchimento do critério de hiperatividade/impulsividade somente. E aqui, enfatiza-se que os subtipos são determinados levando em conta os últimos seis meses. 
Outro ponto notável é o fato de que o quadro sintomático do TDAH se modifica na passagem da infância para a adolescência, visto que a hiperatividade é a principal expressão do transtorno ao longo dos anos pré-escolares, mas é reduzida na adolescência e passa a se enquadrar como nervosismo, inquietude ou impaciência (APA, 2014). Whitbourne e Halgin (2015) explicam que adolescentes com TDAH são essencialmente imaturos $\mathrm{e}$ inclinados a possuir diversas dificuldades com comportamentos e com relacionamentos sociais, além de problemas acadêmicos. Porém, em meninas adolescentes, esses sinais costumam possuir uma evidência reduzida, o que torna o diagnóstico mais complicado.

Dentre as principais caraterísticas do TDAH, além da tríade sintomática (desatenção, hiperatividade e impulsividade), em conformidade com o DSM-5 (APA, 2014) e Sadock, Sadock e Ruiz (2017) é necessário mencionar a labilidade emocional, o autoconceito negativo e hostilidade reativa, baixa tolerância à frustração, desmoralização, déficits cognitivos incluindo a memória, a atenção e a função executiva.

Em vista disso, é nítido o reconhecimento dos desafios enfrentados por pessoas com o referido transtorno do neurodesenvolvimento, considerando a vivência de desvantagens no desempenho escolar, bem como problemas psicológicos e sociais (ASSUMPÇÃO JR. e KUCZYNSKI, 2010). O DSM-5 (APA, 2014) afirma que as dificuldades acadêmicas e escolares estão relacionadas ao sintoma de desatenção elevada, à medida que a alta hiperatividade e/ou impulsividade está geralmente associada à rejeição social.

Vale explanar também, em concordância com Whitbourne e Halgin (2015), que uma variedade de estudos e pesquisas certificam a substancial influência de fatores genéticos. Contudo, a determinação do nível de comprometimento e comorbidade abrange diversos fatores psicológicos e socioculturais, como os efeitos das relações escolares, familiares e com pares.

A partir disso, salienta-se que Assumpção Jr. e Kuczynski (2010) compreendem a cronicidade do problema, mas evidenciam a possibilidade de controle existente, que envolve, sobretudo, a utilização de medicamentos. Desse modo, é importante destacar que o tratamento farmacológico do TDAH, em crianças e adolescentes, é essencialmente constituído pelo uso de estimulantes do SNC, sendo que a maior parte das medicações tem como base o metilfenidato. E dentre estas, cita-se: Ritalina, Ritalina LA, Concerta, Metadate CD, Metadate ER (WHITBOURNE e HALGIN, 2015).

Quanto a isso, ressalta-se, em consonância com Sadock, Sadock e Ruiz (2017) a eficácia do metilfenidato em até três quartos das crianças que possuem o transtorno, com uma quantidade pequena de efeitos colaterais que englobam, usualmente, dores de cabeça, dores de estômago, náusea e insônia. Aqui, cabe informar também sobre o efeito-rebote que alguns pacientes experimentam por um passageiro momento com o fim do efeito da medicação, o que desencadeia notavelmente uma ligeira hiperatividade e uma pequena irritabilidade, e consta, que o uso de simpatomiméticos a curto prazo induz sensação de euforia, desenvolvendo-se tolerância tanto para o sentimento de euforia como também para atividade simpatomimética e concordantemente, Pastura e Mattos (2004) aponta como efeitos colaterais a longo prazo e alerta cuidados com a dependência, efeitos cardiovasculares e possível redução de estatura.

Ademais, Sadock, Sadock e Ruiz (2017) também frisam que deve existir a monitoração do tratamento com estimulantes. Antes do início do uso, a American Academy of Child and Adolescent Psychiatry orienta a realização de exame físico, bem como a verificação da pressão arterial, frequência cardíaca, peso e altura. Esse 
acompanhamento precisa ocorrer a cada três meses, e o exame físico é feito anualmente.

Entretanto, ainda em consonância com os referidos autores, quando se comprova a ineficiência do metilfenidato, são prescritos a dextroanfetamina e combinações de dextroanfetamina e sal de anfetamina, considerados medicamentos no qual devido a necessidade de aumento de dose para atingir efeito, causam tolerância, dependência física e psíquica. Outro tratamento alternativo, de acordo com Whitbourne e Halgin (2015), é a utilização de antidepressivos, incluindo o uso de bupropriona, pemolina, atomoxetina e imipramina.

Diante do exposto, constata-se a importância dos medicamentos no tratamento do Transtorno de Déficit de Atenção/Hiperatividade, visto que os mesmos reduzem seus principais sintomas e auxiliam na melhora da autoestima de crianças e adolescentes (SADOCK, SADOCK e RUIZ, 2017). Todavia, segundo Assumpção Jr. e Kuczynski (2010), a esfera farmacológica não pode ser classificada como intervenção única e suficiente, pois não abarca todas as necessidades terapêuticas de crianças e adolescentes com TDAH, que são satisfeitas apenas com um trabalho multidimensional. Assim, o tratamento psicológico é indispensável para o progresso desses pacientes.

\section{TRANSTORNOS DE ANSIEDADE}

Os transtornos de ansiedade constituem um dos grupos de doenças psiquiátricas mais comuns e predominantes na população, possuindo, como prevalência global em 12 meses, uma taxa de 18,1\% (WHITBOURNE e HALGIN, 2015).

Esta categoria, em conformidade com o DSM-5 (APA, 2014, p.189), diz respeito à transtornos mentais que configuram o medo e a ansiedade como característica fundamental. Em vista disso, é relevante diferenciar os dois conceitos, compreendendo medo como "resposta emocional a ameaça iminente real ou percebida" e definindo ansiedade pela "antecipação de ameaça futura".

No que se refere a etiologia, Sadock, Sadock e Ruiz (2017) consideram a correlação existente entre a genética e fatores socioculturais, ressaltando que traumas e estresses devem ser ponderados. Além disso, Assis et al (2007) declaram que o transtorno de ansiedade na infância pode estar relacionado à fatores familiares, já que as relações entre crianças e cuidadores contribuem para a formação do próprio padrão de funcionamento interno diante às circunstâncias da vida.

Outro ponto importante, segundo Whitbourne e Halgin (2015), diz respeito à necessidade de avaliação acerca da intensidade e persistência do medo e ansiedade, já que estes precisam ser necessariamente excessivos para a realização do diagnóstico. Considera-se, também, que os transtornos dessa categoria divergem entre si devido ao tipo de objeto ou situação que desencadeia os dois principais sintomas.

Desse modo, os transtornos de ansiedade incluem: o transtorno de ansiedade de separação, mutismo seletivo, fobia específica, transtorno de ansiedade social, transtorno de pânico, agorafobia, transtorno de ansiedade generalizada, transtorno de ansiedade induzido por substância/medicamento, transtorno de ansiedade devido a outra condição médica, outro transtorno de ansiedade especificado e transtorno de ansiedade não especificado (APA, 2014).

Tendo isso em vista, de acordo com Knapp (2004), as manifestações dos transtornos de ansiedade em crianças e adolescentes possui três dimensões: comportamental, fisiológica e cognitiva. A primeira é a mais notória, e consiste em 
comportamentos de fuga e esquiva, incluindo choro, voz e mãos trêmulas, roer unhas, chupar do polegar e até correr.

Já os aspectos fisiológicos, ainda em concordância com Knapp (2004), reproduzem a atividade do SNA (Sistema Nervoso Autônomo) e são compostos por náuseas, vômitos, palpitação, sudorese, sensação de sufocamento ou afogamento, ondas de frio ou calor, entre outros. Enquanto a terceira dimensão, a cognitiva, inclui pensamentos ansiosos, como por exemplo, preocupações sobre o que acontecerá e sobre o julgamento dos outros.

Diante do exposto, é possível reconhecer que o transtorno de ansiedade em crianças e adolescentes possui diversas consequências negativas, e dentre elas, são reveladas dificuldades em realizar trabalhos escolares ou profissionais, problemas nos relacionamentos sociais (com amigos e familiares) e incapacidade de apreciar momentos de lazer (WHITBOURNE e HALGIN, 2015).

Portanto, o tratamento é indispensável, uma vez que, segundo o DSM-5 (APA,2014), se não for tratado, o transtorno frequentemente persevera até a idade adulta. Então, em relação à farmacoterapia, as classes de medicamentos utilizados para tratamento de transtornos de ansiedade em crianças e adolescentes, segundo Maia e Rohde (2009, s/p), são os "ISRS (fluoxetina, sertralina, paroxetina e fluvoxamina), antidepressivo tricíclico (imipramina) e benzodiazepínicos (alprazolam e clonazepam)".

É necessário, entretanto, prestar atenção nos efeitos colaterais dessas medicações, com um cuidado ainda mais especial pelo fato de serem crianças e adolescentes. Assim, Sadock, Sadock e Ruiz (2017) explicam que os ISRS usualmente causam hipotensão postural, boca seca (geralmente no uso de paroxetina), náuseas, vômitos, dispepsia, tremores, dores de cabeça (no uso de fluoxetina), bocejo não relacionado à fadiga ou pobreza do sono noturno, "cegueira emocional" (uso crônico) e sudorese.

O referido autor segue admitindo que a imipramina possui como efeitos adversos: sedação, efeitos anticolinérgicos, hipotensão postural, convulsões e anormalidades da condução cardíaca. E os benzodiazepínicos podem causar no paciente: sedação, fadiga, perdas de memória, sonolência, prejuízos na coordenação motora, diminuição da atenção, da concentração e dos reflexos, bem como tolerância, dependência e abstinência, sendo necessária a avaliação ininterrupta como parte do tratamento de pacientes com uso desse fármaco.

\section{TRANSTORNOS DISRUPTIVOS, DO CONTROLE DE IMPULSOS E DA CONDUTA}

Primeiramente, é importante apresentar, em consonância com o DSM-5 (APA, 2014), que os transtornos disruptivos, do controle de impulsos e da conduta dizem respeito à uma categoria que possui como característica substancial a grande dificuldade no controle de emoções e comportamentos, sendo que esta é expressada com a violação dos direitos dos outros e/ou com significativos atritos com regras sociais e figuras de autoridade.

Aqui, de acordo com Sadock, Sadock e Ruiz (2017) e o DSM-5 (APA, 2014), cabe esclarecer que esses aspectos ocorrem, em alguma proporção, durante o processo de desenvolvimento e crescimento das pessoas, e por isso, é de suma relevância previamente analisar e compreender a frequência, a intensidade e a gravidade desses comportamentos, levando sempre em conta a idade, o gênero e a cultura a qual o indivíduo está inserido. 
Além disso, segundo o DSM-5 (APA, 2014), é durante a infância ou a adolescência que os referidos transtornos propendem a se iniciar. Considera-se, também, que os diagnósticos são comumente mais atribuídos a pessoas do sexo masculino, existindo uma grande possibilidade de comorbidade.

Tendo isso em vista, destaca-se que os transtornos disruptivos, do controle de impulsos e da conduta abrangem oito transtornos (WHITBOURNE e HALGIN, 2015). Entretanto, aprofunda-se, no presente trabalho, o transtorno de oposição desafiante e o transtorno de conduta.

O transtorno de oposição desafiante é reconhecido fundamentalmente pela existência de humor irritável/raivoso, comportamento questionador/desafiante e índole vingativa no decorrer de seis meses. Os sintomas são geralmente revelados, de forma notória, em relações com pessoas mais íntimas, e desse modo, podem se manifestar em um ambiente apenas, no qual habitualmente é a casa (DSM-5, 2014; SADOCK, SADOCK e RUIZ, 2010).

De acordo com Whitbourne e Halgin (2015, p. 249), as crianças e adolescentes que possuem esse transtorno são "suscetíveis, ressentidos, agressivos, rancorosos e donos da verdade". Todavia, Sadock, Sadock e Ruiz (2017) expõem que esses indivíduos não se auto avaliam com tais características e defendem que os comportamentos são consequências de acontecimentos injustos.

Posto isto, é indispensável destacar que o transtorno de oposição desafiante desencadeia sofrimento para o sujeito, bem como para os familiares e pessoas próximas. Também prejudica, independentemente da inteligência, o desempenho escolar, acadêmico, profissional e as relações sociais, e como consequência, é possível identificar baixa autoestima, baixa tolerância a frustração, sensação de inadequação, humor deprimido e acessos de raiva. (WHITBOURNE e HALGIN, 2015; SADOCK, SADOCK e RUIZ, 2017).

O transtorno de conduta, por sua vez, em conformidade com o DSM-5 (APA, 2014), é caracterizado por constantes comportamentos, nos últimos 12 meses, que resultam em violação de direitos dos outros e normas já compreendidas pela idade. Esse padrão é manifestado em vários ambientes, com agressão a pessoas e animais, destruição de propriedade, falsidade ou furto, e/ou violações graves de regras.

À vista disso, convém compreender que os sintomas não surgem instantaneamente, e dessa maneira, os mesmos passam por um processo de evolução da gravidade até o desenvolvimento de um persistente quadro comportamental que engloba a violação dos direitos dos outros (SADOCK, SADOCK e RUIZ, 2017).

Ademais, salienta-se, consoante a Whitbourne e Halgin (2015) e ao DSM-5 (APA,2014), a necessidade de determinação do subtipo, o qual leva em consideração o início do problema. Quando ao menos um sintoma é expressado antes dos 10 anos, o transtorno de conduta é classificado como "tipo com início na infância"; quando nenhum sintoma é manifestado antes dos 10 anos, diagnostica-se "tipo com início na adolescência"; e se não há disponibilidade de informações para identificar o começo, é declarado "início não especificado".

Dentre as principais características de personalidade, frisa-se, em concordância com o DSM-5 (APA,2014), que as crianças e adolescentes com transtorno de conduta, mesmo projetando uma imagem de dureza, possuem baixa autoestima, baixo autocontrole e baixa tolerância a frustrações, além de serem muito irritáveis, desconfiados, insensíveis a punições, imprudentes e costumam frequentemente possuir explosões de raiva. 
Sadock, Sadock e Ruiz (2017) complementam admitindo que não há esforços para ocultar os comportamentos ilegais e que atos ou pensamentos suicidas são usuais, uma vez que os prejuízos sociais e interpessoais são perceptíveis, incluindo afastamento da escola e trabalho, doenças sexualmente transmissíveis, danos físicos ocasionados por acidentes ou brigas, prisão, entre outros.

Por fim, é interessante informar que, segundo o DSM-5 (APA, 2014, p.473) "o transtorno de oposição desafiante é um percursor comum do transtorno da conduta do tipo com início na infância". Porém, muitas crianças e adolescentes que possuem o transtorno de oposição desafiante não desenvolvem posteriormente o transtorno de conduta, e conforme Sadock, Sadock e Ruiz (2017), cerca de um quarto delas não preenche os critérios diagnósticos para TOD com o passar do tempo.

Então, acerca do prognóstico, diversos fatores devem ser considerados, inclusive o tratamento adequado. Sendo assim, frisa-se que, de acordo com Teixeira (2006), o transtorno de oposição desafiante ainda não possui um tratamento medicamentoso específico. Porém, é possível encontrar diferentes relatos em artigos e trabalhos científicos sobre a utilização de psicofármacos objetivando o controle dos sintomas opositivos e desafiadores.

Dentre as medicações usadas, Serra-Pinheiro et al (2004) e Sadock, Sadock e Ruiz (2017) garantem que as mais eficazes, quando se trata de instabilidade de humor observável, são o ácido valpróico, haloperidol (Haldol), risperidona (Risperdal) e lítio (Eskalith), utilizados tanto para o tratamento do transtorno de oposição desafiante como para o transtorno de conduta.

Em concordância com Stucchi (2009), o primeiro agente farmacológico possui como efeitos colaterais: dor abdominal, náusea, vômito, diarreia, vertigem, dor de cabeça, convulsão, reflexos aumentados, prejuízo na memória, edema, pele seca, boca seca, incontinência urinária, entre outros. Já o haloperidol é um antipsicótico típico, altamente sedativo, e por isso, pode causar cansaço e apatia nos pacientes. Além disso, ele possui efeitos indesejáveis, os sintomas extrapiramidais, que correspondem a diversos transtornos motores, incluindo, após alguns anos, a discinesia tardia (WHITBOURNE E HALGIN, 2015).

A risperidona tem como efeitos adversos mais comuns, em crianças, a sonolência, cefaleia e ganho de peso (TEIXEIRA, 2006). E por fim, Stucchi (2009) também afirma que o uso de lítio pode desencadear efeitos secundários como: aumento da sede, aumento da excreção urinária, sensação de enjoo ou náusea, dores ligeiras do estômago, ligeiro tremor das mãos, sonolência, enfraquecimento muscular, diminuição da capacidade ou interesse sexual, tontura, boca seca, aumento de peso, sabor metálico e predisposição para acne ou psoríase.

Para o transtorno de conduta, Freitas e Catelan-Mainarde (2013) confirmam que a carbamazepina (Tegretol) também pode ser prescrita, uma vez que ela auxilia na redução da agressividade. Contudo sua utilização pode comumente causar tontura, sonolência, náusea, vômito e visão turva (STUCCHI, 2009).

\section{CONSIDERAÇÕES FINAIS}

A psicopatologia na infância e adolescência se constitui como um tema de grande relevância nos dias atuais, tendo em vista que estudos demonstram uma alta prevalência de transtornos psicopatológicos nestas fases do desenvolvimento, e esta temática engloba diversas áreas do conhecimento, como a médica, farmacêutica e psicológica. 
Logo, o transtorno de déficit de atenção e hiperatividade, assim como os transtornos pertencentes às categorias dos transtornos de ansiedade e dos transtornos disruptivos, do controle de impulsos e da conduta, caracterizam-se como algumas das psicopatologias de manifestação mais comum durante a infância e a adolescência.

Assim, partindo do pressuposto de que o ser humano deve ser compreendido a partir de uma visão biopsicossocial, destaca-se a importância de observar o contexto em que se encontram estas crianças e adolescentes e considerar os inúmeros fatores que influenciam para ocasionar e manter estes transtornos.

Concernente a isso, é importante ressaltar que a medicalização se faz necessária em determinados casos, no entanto, o tratamento medicamentoso atende somente às necessidades biológicas do indivíduo. Desta maneira, estudos demonstram que um tratamento combinado de psicoterapia e farmacoterapia possibilita uma melhora mais efetiva nos quadros de psicopatologias, pois assim é possível atender também às questões psicológicas e sociais envolvidas no caso.

Portanto, compreende-se que a preocupação com a saúde mental infantil pode ser considerada relativamente recente, havendo muitas questões a serem pensadas e elaboradas sobre este assunto, que se configura como um dos desafios éticos e políticos da saúde pública brasileira.

\section{REFERÊNCIAS}

AMERICAN PSYCHIATRIC ASSOCIATION (APA). Manual diagnóstico e estatístico de transtornos mentais: DSM-5. 5. ed. Porto Alegre: Artmed, 2014.

ASSIS, S. G. de; XIMENES, L. F.; AVANCI, J. Q.; PESCE, R. P. Ansiedade em crianças: um olhar sobre transtornos de ansiedade e violências na infância. Rio de Janeiro: FIOCRUZ/ENSP/CLAVES/CNPq, 2007. Disponível em: < http://docs.wixstatic.com/ugd/7ba6db 319224594ed14b0e89e97401ccce8ed4.pdf > Acesso em: 02 jun. 2019.

ASSUMPÇÃO JUNIOR, F. B.; KUCZYNSKI, E.; e cols. Qualidade de vida na infância e na adolescência: orientações para pediatras e profissionais de saúde mental. Porto Alegre: Artmed, 2010.

CANGUILHEM, G. O Normal e o Patológico. 6ª ed. Rio de Janeiro: Forense Universitária, 2010.

FREITAS, M. A. L. de; CATELAN-MAINARDES, S. C. Estudo dos transtornos de comportamento disruptivo na infância e adolescência pela perspectiva da neurobiologia e ações medicamentosas. In: Anais do VII EPCC - Encontro Internacional de Produção Científica Cesumar, 2013, Maringá. Maringá: Centro Universitário Cesumar (UNICESUMAR), 2013. Disponível em:

$$
\text { http://www.cesumar.br/prppge/pesquisa/epcc2013/oit mostra/Marina Aparecida Lui }
$$
$\underline{z}$ de Freitas.pdf $>$ Acesso em: 09 jun. 2019.

SADOCK, B. J.; SADOCK, V. A.; RUIZ, P. Compêndio de Psiquiatria: ciência do comportamento e psiquiatria clínica. 11. ed. Porto Alegre: Artmed, 2017. 
KNAPP, P. Terapia cognitivo-comportamental na prática psiquiátrica. Porto Alegre: Artmed, 2004.

MAIA, C. R. M.; ROHDE, L. A. Psicofármacos para o tratamento de transtornos de ansiedade em crianças e adolescentes: uma revisão sistemática. Revista Brasileira de Psiquiatria. São Paulo, vol. 29, n. 01, p. 72-79. Mar, 2007. http://dx.doi.org/10.1590/S1516-44462006005000019.

SERRA-PINHEIRO, M. A.; SCHMITZ, M.; MATTOS, P.; SOUZA, I. Transtorno desafiador de oposição: uma revisão de correlatos neurobiológicos e ambientais, comorbidades, tratamento e prognóstico. Revista Brasileira de Psiquiatria. São Paulo, vol. 26, n. 4, p. 273-276. Dez, 2004. http://dx.doi.org/10.1590/S151644462004000400013

STUCCHI, H. K. de O. Como tratar crianças e adolescentes com transtornos psiquiátricos e o papel da família no tratamento. São Paulo: Cruzeiro do Sul Online, 2009. Disponível em:

$<$ http://abp.org.br/portal/clippingsis/exibClipping/?clipping=9349 > Acesso em: 27 ago. 2017.

TEIXEIRA, G. Terapêutica Medicamentosa no Transtorno Desafiador Opositivo: revisão da literatura. Arquivos Brasileiros de Psiquiatria, Neurologia e Medicina Legal. Rio de Janeiro, vol. 100, n. 02, p. 55-58. Abr/Maio/Jun, 2006. Disponível em: < http://www.comportamentoinfantil.com/tdo1.pdf > Acesso em: 06 jun. 2019.

TISSER, L. Transtornos psicopatológicos na infância e na adolescência. Novo Hamburgo: Editora Sinopsys, 2018.

WHITBOURNE, S. K.; HALGIN, R. P. Psicopatologia: perspectivas clínicas dos transtornos psicológicos. 7.ed. Porto Alegre: AMGH, 2015. 